\title{
Blending multi-source evapotranspiration datasets via triple collocation approach
}

\author{
Jongjin Baik ${ }^{1}$, Jongmin Park $^{2}$, and Minha Choi ${ }^{1}$ \\ ${ }^{1}$ Sung Kyun Kwan University \\ ${ }^{2}$ University of Maryland at College Park
}

November 6, 2020

\begin{abstract}
In this research, we provided a framework to merge well-known satellite- and reanalysis-based global ET products (GLDAS, GLEAM, MOD16, and MERRA) using the triple collocation (TC) along with least-square based merging scheme without the utilization of high-quality ground measurement over East Asia. Firstly, the error characteristics of each ET product were statistically estimated using TC metrics with four different combinations. Results revealed that GLEAM showed the least error variance and highest product-truth correlation coefficient for most land cover types, followed by GLDAS, MERRA, and MOD16. TC-based error characteristics at different land cover types were reflected to parameterize weighting factors for individual ET products, and in turn, utilized in producing the merged ET estimates. Evaluation of merged ET estimates was conducted at 11 flux tower sites located within the study area. When relatively low-quality ET products (MERRA and MOD16) were used as input for TC metric, the accuracy of the merged ET estimates was better than those of the two individual ET products at all three land cover types. Furthermore, when two relatively high-quality ET products (GLEAM and GLDAS) were used as input for TC, the accuracy of merged ET estimates were greater than that of GLEAM and showed the highest statistical performance among the ET products over the three land cover types. Merged ET estimates from scenarios containing GLEAM and GLDAS showed MAE (RMSE) ranging from 0.275 to $0.692 \mathrm{~mm} / 8$ day ( 0.399 to $0.873 \mathrm{~mm} / 8$ day) and correlation coefficient ranging from 0.864 to 0.933 in compared to in-situ measurements. These statistics showed substantial improvement when compared to the original ET products (MAE: 0.327 to $1.172 \mathrm{~mm} / 8$ day, RMSE: 0.464 to $1.455 \mathrm{~mm} / 8$ day, and correlation coefficient: 0.636 to 0.925$)$ over the three land cover types. These results confirmed that a TC-based merging framework could enhance the accuracy of terrestrial ET.
\end{abstract}

\section{Hosted file}

manuscript_v1.pdf available at https://authorea.com/users/373735/articles/491339-blendingmulti-source-evapotranspiration-datasets-via-triple-collocation-approach

\section{Hosted file}

Figure.pdf available at https://authorea.com/users/373735/articles/491339-blending-multisource-evapotranspiration-datasets-via-triple-collocation-approach

\section{Hosted file}

Table.pdf available at https://authorea.com/users/373735/articles/491339-blending-multisource-evapotranspiration-datasets-via-triple-collocation-approach 